\title{
REDISCOVERING FREE ASSOCIATIONS FOR USE IN PSYCHOTHERAPY
}

\author{
LORAND B. SZALAY \\ Chevy Chase, Maryland
}

\author{
JEROME F. X. CARROLL \\ New York, New York
}

\author{
FRANK TIMS \\ National Institute on Drug Abuse \\ Rockville, Maryland
}

Free associations emerge from the present investigations as a rich source of psychological insights useful in therapeutic applications. In light of the early uses of free association by the founding fathers of psychotherapy, Freud and Jung, such application capabilities should come as no surprise. The present approach is enhanced by the progress of empirical semantics and a heavy reliance on computers, yet it relies essentially on realizations similar to those of Freud and Jung in which insights into subjective worlds were revealed in free associations. The present investigations used free associations to reconstruct perceptual and attitudinal dispositions associated with substance abuse to measure changes indicative of the psychological impact of treatment. Associations to words as well as to pictures were used. The results are compared for their information value and relevance to therapeutic applications.

The research reported here is based on a recently completed study conducted under the sponsorship of the National Institute on Drug Abuse. The views expressed here are those of the authors.

Correspondence regarding this article should be addressed to Lorand B. Szalay, Rapport, Inc., 6935 Wisconsin Avenue, Suite 500, Chevy Chase, MD 20815.

\section{Rediscovering Free Associations for Use in Psychotherapy}

Since the early attempts of Freud and Jung to penetrate the inner world of the client through free associations, clinical interest in the client's inner world has remained strong. The perceived value of free associations to penetrate this inner world, however, has gradually diminished. The present investigations provide an opportunity to rediscover the value of free associations as a way to assess systematically the subjective world of clients and thus enhance therapeutic communications and better evaluate treatment progress and outcomes.

Contemporary clinical interest in subjective meanings is evident in the constructs of psychological meanings and cognitive representations which are central to several therapeutic schools, such as logotherapy (Frankl, 1962), rational-cognitive therapy (Ellis, 1962; 1975), and cognitive behavior therapy (Beck, 1976). These constructs are used by clinicians in the attempt to bridge the subjective world of the client with that of his/ her external world. Conflict between these two worlds is recognized as a potentially pathogenic condition. According to Beck (1976), the aim of therapy is to help the client bring his/her private meanings or subjective world in conformity with the meanings of the immediate environment. Sterman (1991) also speaks explicitly of the task involved in reducing the distance between the client's private meanings in his own "representational system" and the public meanings in his/ her external world. Therapists working with the neuro-linguistic programming model use reframing techniques and the negotiation model to 
achieve similar objectives (Bandler \& Grinder, 1982).

Although numerous therapeutic models recognize the conflict between private meanings and the external world, a systematic assessment of such differences generally falls beyond the interest of therapists. The empirical study of subjective meanings has been approached by social psychologists and psycho-linguists as a "system of subjective representation." The concepts of cognitive maps (Tolman, 1948), systems of cognitions (Triandis \& Vassiliou, 1967), meaning system (Osgood, Suci \& Tannenbaum, 1957), cognitive representation (Downs \& Stea, 1973), internal representation (Shepard \& Chipman, 1970), and subjective lexicon (Miller, 1967) converge in the fundamental assumption that people's behavior is organized and guided by their subjective meanings, by a system of subjective representation.

The development of specialized analytic techniques in this field of study has resulted in new capabilities to assess subjective meanings on a solid empirical foundation. Osgood (1957), a pioneer in this field, developed an ingenious analytic tool, the semantic differential, for the "measurement of meaning." The theoretical rationale of using word associations in the empirical study of meanings has its roots in the work of Noble (1952) and Deese (1965). Following this same line of theoretical orientation and objectives, Szalay and his associates developed the Associative Group Analysis (AGA) (Szalay \& Brent, 1967). As described in the monograph Subjective Meaning and Culture (Szalay \& Deese, 1978), the AGA method is based on a representational model of behavioral organization and relies on continued free associations to reconstruct the internal world and subjective meanings of people that are inaccessible to more direct methods.

To reconstruct the representational systems of subjective meanings with the AGA method requires the analysis of hundreds of thousands of free associations to selected key themes elicited from medium size samples $(N=50-100)$. The strategy is to map subjective worlds by using computerized analytic methods focused on the main dimensions of behavioral organization. In contrast to most direct and structured methods of psychological assessment, this approach does not ask questions or use scales. Rather, it infers what is dominant or of negligible importance in people's subjective worlds from the spontaneous distributions of their free associations.
The AGA method is used in the present investigations to explore the inner world of drug addicts before and after treatment and to reconstruct their psychological dispositions associated with substance abuse. Contemporary clinical research in the field of substance abuse has tended, however, not to focus on the internal subjective world of drug users but rather to examine the dimensions of drug use through the evaluation of personality traits. This approach followed, rather naturally, from the high status of personality constructs in psychology, even though the inconclusive findings of the research have been disappointing. Locus of control, for example, was found to correlate significantly with substance abuse in some studies (Craig, 1986; Zeiner et al., 1985; Krampen \& Von Eye, 1984; Jurich \& Polson, 1984; Weidman, 1983; Segal \& Merenda, 1975) but not in others (Doherty \& Matthews, 1988; Dielman et al., 1987; Des Jarlais, 1986; Olton, 1985; Malvin et al., 1985; Rohsenow, Smith \& Johnson, 1985; Biggs, Bender \& Foreman, 1983; McBride, 1982). McBride (1982) suggested that such contradictory results may be due to test settings which elevate social desirability and thereby inflate internality scores. Des Jarlais (1986) found that treatment represented a reassertion of lost control for heroin addicts, suggesting that locus of control is situationally influenced.

In a recent review of personality concepts and their relationship to addiction, Sutker \& Allian (1988) concluded that the etiology, expression, and consequences of substance abuse require broader conceptualizations that encompass biological and sociocultural dimensions in addition to personological dimensions. While researchers vary in their conclusions, they show a similar trend in shifting their focus of attention away from personality constructs.

Rather than approaching the psychological dimensions of drug use through personality constructs, our approach is to focus on the identification and mapping of the perceptual and evaluative dimensions of cognitive organization. Our research in recent years has shown the analytic sensitivity of the Associative Group Analysis (AGA) method in the assessment of perceptual and motivational dispositions that differentiate drug users from non-users: e.g., college students who regularly use drugs (primarily marijuana) and college students of matching sociodemographic background who do not use drugs (Szalay, 1990). Significant differences were found in the domains 
of drugs, self-image, relationship to family and friends, and general human problems. Similar findings were obtained in the comparative study of high school drug user and non-user students from three different geographic areas of the United States (Szalay et al., 1992).

Once it was shown that the analytic measures could differentiate between drug users and nonusers and between users of different levels of drug use as well, we tested whether the analytic approach may show consistent differences between drug addicts starting treatment and those completing treatment. In a pilot study of 60 pretreatment and 60 post-treatment clients (Szalay et al., in press), the differences were significant and interpretable by relating the differences found in the internal states of these treatment populations to the effects of the treatment process. A later study of larger client populations of a therapeutic community (Szalay, in press) confirmed the findings on perceptual and motivational changes $o c$ curring during the treatment process. The results supported the feasibility of developing an analytic instrument of process evaluation which could provide insights into internal states indicative of treatment success. The investigations reported here pursue similar objectives by using word- and picture-stimulated free associations to identify perceptual and motivational trends of pre-treatment and post-treatment clients.

In contrast to the generally negative or contradictory research results obtained on the relationship of personality constructs and drug use, the AGA studies support the conclusion that psychological dispositions associated with drug abuse can be empirically identified. The results have shown an unsuspected close relationship between drug behavior and internal states in certain dimensions of behavioral organization.

Our focus of interest is the perceptual/motivational variables that affect behavior below the level of conscious awareness. The representational model of behavioral organization relies on psychological dispositions characterized by dominant perceptions and motivations. In this model, human behavior hinges not on the balance of objective costs and benefits but on perceived costs and perceived benefits. These values are determined by the individual's subjective world, the subjective system of representation of the environment, which is generally too private, too personal to be accessible. Based on this model of representational organization of behavior, we as- sume that the individual's choices are predictable, granted that the dominant perceptions and motivations-for example, to use drugs or not to use drugs - can be assessed.

\section{Method}

\section{Subjects}

The research is based on a cross-sectional comparison of pre-treatment and post-treatment clients who resided in two urban-based therapeutic communities. The therapeutic community (TC) is a treatment modality, used at long-term, residential treatment facilities, which emphasizes the following factors: 1 ) the importance of role models (e.g., recovery staff) to promote positive behavioral changes, 2) involvement of peers in recovery process, 3) emphasis on work, 4) emphasis on assuming personal responsibility for one's behavior and consequences, and 5) instillment of a sense of concern for community as a whole (DeLeon, 1986; DeLeon \& Rosenthal, 1989).

Those who enter the therapeutic community are hardcore users predominantly of cocaine and crack. They suffered from serious behavioral difficulties: the inability to hold jobs or earn a living, to function in normal family roles, to meet personal obligations. Most of the addicts entered treatment after reaching a level of dependency that forced them to seek treatment. The ages of the clients ranged from 19 to 44 , with an average age of 29.

The pre-treatment group consisted of 100 habitual drug users who had been in the therapeutic community less than a month and were at the beginning of their treatment process. This group was $73 \%$ male and $27 \%$ female, and the ethnic makeup of the sample was $22 \%$ white, $60 \%$ black, and $18 \%$ Hispanic. Fifty-three percent of the clients had been in drug treatment before.

The post-treatment group was formed of 100 residents from the same treatment facilities, who had been there an average of 14 months. They were judged to be successfully treated by several criteria, such as maintaining a drug-free status over many months, assuming increasingly demanding jobs and responsibilities within the treatment center and later on in normal job settings, developing plans, holding to schedules, developing personal ties, etc. This group was $55 \%$ male and $45 \%$ female, and the ethnic make up of the sample was $21 \%$ white, $68 \%$ black, $10 \%$ 
Hispanic, and $1 \%$ American Indian. In the posttreatment group, $30 \%$ of the clients had previously been in drug treatment.

\section{The Associative Group Analysis Method}

The data collection relied on ten selected word themes and thirty selected pictures representing people and social interaction scenes to elicit multiple free associations. The AGA word and picture association task was presented as a simple paperand-pencil language task rather than an attempt to probe personal beliefs or attitudes. The pretreatment and post-treatment respondents received a booklet with a short background questionnaire and the stimulus themes (e.g., me) printed twelve times on separate slips of paper. They were asked to write as many ideas related to each presented theme as they could think of in one minute. Thirty pictures were then presented, one by one, on a slide projector, and the respondents were again given one minute to respond in the same manner.

Respondents generally give six to eight different associations for each theme word or picture. Research has shown that the first responses are slightly more informative on the salient subjective meaning than the ones that follow. The strategy for deriving the scores empirically was to retest with the same free association task after one week and measure the percentage of first test responses at each rank place which recurred in the retest of the same subjects. The stability of responses is indicated by the results obtained for rank place of emission and mean percentage of stability, respectively: first, .60; second, .48; third, .42; fourth, .34; fifth, .32; sixth, . 30 ; seventh, .25; eighth, .20; ninth, .15; and tenth, .11 (Szalay \& Deese, 1978). On that basis the weights assigned to each response, from first to last, are: $6,5,4$, $3,3,3,3,2,2,1,1,1$. The weighting scores thus express the relative salience and stability of each response given.

Response lists illustrate the distribution of responses, revealing the main mosaic pieces of the respondent's subjective perceptions and evaluations. Each response has a score value that reveals how salient a particular idea or attribute is as a mosaic element of the individual's subjective representation of a particular theme or image. The response distributions provide insights into the subjective worlds of the respondents. Past experience has shown that every shared response offers a mosaic piece of information; the response score indicates how important each piece of information is in reconstructing a group's subjective image.

Because the mosaic pieces revealed by the response distributions are many and frequently related, content analysis is used to group the responses into relevant clusters or categories. Responses conveying negative affects are placed into clusters labeled by the most salient reactions; references to positive affects are placed in clusters containing related responses. Such clusters reveal major response trends which inform on dominant perceptions and evaluations. Past studies have shown that such categorizations or content analysis can be performed with a reasonable degree of reliability. The mean correlation between analysts working independently was .7 (Szalay \& Deese, 1978).

\section{Measures}

Several analytic measures are applied to gauge the organization of the system of subjective representations of the pre-treatment and post-treatment clients along the main dimensions of perceptions, dominance, and evaluations.

Subjective views, perceptions. The similarity of subjective views and perceptions of a particular theme for different groups is measured by comparing the distributions of their free assocations. Pearson's product-moment correlation is used. For groups, the reliability of the perceptual similarity measure based on split-half comparison of over 40 themes was 82 (Szalay \& Bryson, 1973). Perceptual similarity scores can also be computed for each individual with reference to the distributions of free associations. For each stimulus word, responses which differentiated the groups (e.g., pre-treatment and post-treatment clients) are identified. Each such response is scored +1 if it is more characteristic of one group (e.g., post-treatment clients), and -1 if it is more characteristic of the other group (e.g., pre-treatment clients). In this manner, individual perceptual similarity scores are calculated to reflect the degree to which a subject's perceptual trends are more similar to pre-treatment or post-treatment clients.

Priorities, subjective importance. The importance or dominance of a particular stimulus theme to a particular person or group is inferred from the number of responses offered in the association task. The dominance scores calculated both on an individual and group basis are analogous to Noble's (1952) widely tested measure of "meaningfulness." The group dominance scores have 
been used to measure differences between groups in their subjective priorities, as well as to trace changes in priorities over time. The reliability of the group dominance score $(r=.93)$ was measured by test-retest comparison (Szalay \& Bryson, 1973). Individual dominance scores are computed as the number of responses given to each stimulus theme. Through a discriminant function procedure the dominance similarity score is calculated on the basis of discriminant function coefficients for the individual dominance variables. It indicates the extent to which a respondent's pattern of dominance is more similar to pre-treatment or post-treatment clients.

Attitudes, evaluations. Attitudes, positive versus negative evaluations, are the most widely researched and recognized psychological variables. One of the ways to reconstruct how a person or group evaluates a particular issue or theme without asking direct questions is to assess the predominance of positive versus negative responses produced by the theme in continued free association tasks. Evaluative scores are also calculated on an individual basis. The list of responses to all the stimulus words are reviewed by two judges. The two judges rate each response word in terms of its positive or negative affect (interjudge $r=.95$ ). For each subject the evaluation of each stimulus theme is computed as the sum of the judges' evaluation of the response words. An evaluative similarity score is constructed following the same rationale as the dominance similarity score. It is calculated on the basis of discriminant function coefficients for the individual evaluative variables. It indicates the extent to which the respondent's pattern of evaluations is more characteristic of pre-treatment or post-treatment clients.

\section{Validity of the Method}

The novelty and richness of free associations, the quantitative nature of the data, and their potential use in various diagnostic and therapeutic applications make it particularly compelling to address questions related to its validity. Validation is naturally a complex task with respect to a method that pursues the ambitious objective of mapping systems of subjective representation or cognitive organization. Information produced on the psychological dispositions of drug users ranges from specific perceptions and attitudes to the organization of their systems of subjective representations and their behavioral implications.
Several sourcs of information related to validity are considered below.

Validation of inferences on selected variables. In past investigations the AGA-based findings on select psychological variables, particularly subjective meanings and attitudes, have been compared with findings obtained through other independent measures. AGA-based data showed high correlations with semantic differential (word-adapted) $(r=.60)$, similarity judgment $(r=.73)$, judgment of relationship ( $r=$ $.77)$, and semantic grouping $(r=.84)$ (Szalay \& Bryson, 1974). AGA-based attitude measures showed extremely high correlations with semantic differential attitude scales $(r=.93)$ (Szalay \& Brent, 1967) and multiple-choice attitude questionnaires $(r=.9)$ (Szalay, Windle \& Lysne, 1970).

Behavioral validation. In previous studies we tested the accuracy of using free association based perceptual and attitudinal data to identify various categories of drug users and non-users. In a comparative study of 120 clients in a therapeutic community setting (60 who had just started treatment and 60 who had completed treatment), $85 \%$ were correctly identified through discriminant analysis of the perceptual, evaluative, and dominance data based on 24 themes (can. corr. $=.97, \mathrm{WL}=$ $.0658, d f=72, p<.002$ ) (Szalay et al., in press).

The strategy was also applied in a socialization study to a larger treatment population of 400 clients to show the percent of pre-treatment and post-treatment clients correctly classified: $87 \%$ based on the perceptual measure (can. corr. = .75 , chi-square $=327.3, d f=1, p<.000$ ), $75 \%$ based on the dominance measure (can. corr. $=.56$, chi-square $=140.4, d f=40, p<.000$ ), and $77 \%$ based on the evaluative measure (can. corr. $=.62$, chi-square $=187.6, d f=40, p<$ .000) (Szalay, in press). In a split-half analysis of this sample, the correlation between the perceptual similarity measure and pre/post status was .85 within group and .66 across groups, indicating a very strong relationship between psychological dispositions and behavior.

In some studies we relied on self-reported drug use as a criterion for gauging the accuracy of identification of users and non-users based on free association data reflecting their psychological dispositions. In a study of $\mathbf{4 0 0}$ college students (Szalay et al., in press) the results showed $80 \%$ of the users and $74 \%$ of the non-users were correctly 
classified based on discriminant analysis of the perceptual (across-group), evaluative, and dominance data on 24 themes (can. cor. $=.63$, chisquare $=169.68, d f=49, p<.000$ ). In another study of $\mathbf{3 0 0}$ high school students multiple correlation of the perceptual, evaluative, and dominance measures (across-group) with self reported drug usage was .56 (chi-square $=75.46, d f=$ $3, p<.001$ ) (Szalay et al., 1992).

Our association-based research findings are in agreement with various clinical studies in showing that drug use is commonly associated with poor self-image (Biase, 1984; Carroll, 1982; Kaplan, 1982), strained social relations (Botvin \& McAlister, 1982; Kandel, 1980), and alienation (Craig, 1988; Burr, 1984; Stein, Soskin \& Korchin, 1975). While this consistency is encouraging, the value of the AGA approach appears to be greater in obtaining new insights rather than confirming existing knowledge.

\section{Results}

Due to the limited space of this article, results are presented only on the associations to one word (drugs) and on one picture (\#918-Two men walking). Trends in the responses of the pre-treatment and post-treatment clients are presented. The discriminant function analysis is shown to correctly identify and differentiate between the two groups.

As can be seen in Figure 1, the pre-treatment clients think of drugs in very concrete, specific terms. They name specific drugs (crack, marijuana, heroin) and have alcohol more saliently in mind. This heavy emphasis on specific substances leaves limited space in the minds of these as yet untreated addicts to consider other things, including the consequences of drugs, harmful effects, crime, etc. The post-treatment clients pay less attention to specific types of drugs and alcohol and they show more concern with harm and negative consquences: killing, destruction, danger. Similarly, they express more negative feelings and attitudes about drugs: bad, crazy, insane, disgust. They think more of treatment and stopping drug use. They also show more concern with the mind-altering effects of drugs (high, escape).

The results across the three words used in the representation of the drug domain (drugs, alcohol, hooked) reveal trends that are summarized in Figure 2. The pre-treatment clients consistently place more emphasis on specific substances.
While they emphasize the social aspects of drugs, the pre-treatment clients also recognize negative factors which include pain and fear. This polarity reflects their recognition of the need for treatment coupled with the pleasurable effects of the abusive substances. On the other hand, the post-treatment clients show an overall trend in the rejection of drugs and the recognition of addiction. Their responses are generally negative with an emphasis on the fear, hate, and danger involved in drug usage. These trends in the drug domain can be related to the self domain where pre-treatment clients express anger and sadness and the posttreatment clients convey a sense of regained confidence and strength in relation to self.

With regard to the pictures, it is only natural to assume that the same picture conveys the same meaning and tells the same story to everybody, but the example shown in Figure 3 demonstrates that this is not the case. The pre-treatment clients saw two men who were unemployed and looking for work. They referred more to the physical aspects of the scene, such as the building, the street, and the people. The post-treatment clients, on the other hand, more strongly identified the two men as friends. They saw them as having problems, but they also thought of helping, coping, and support. The clients who had undergone treatment clearly expressed more awareness of the possibility of solving or coping with problems and relying on friends for help.

The pre-treatment clients registered more specific details and focused on what is directly observable: people, objects, and events. They took note of the actions without evaluating them and mentioned the people without exploring the relationships between them. The post-treatment group went beyond the observable and attempted to understand what was going on. Post-treatment clients expressed more emotions, both positive ones like love and care and negative ones such as pain, confusion, and loneliness. They also showed more preocupation with drugs, particularly in response to pictures in which drug use was not explicit but that the people and events of the pictures reminded them of drugs. When drug or alcohol use was featured in the picture, the pre-treatment clients mentioned it more, probably because they tend to view the pictures more in terms of concrete observable details. When the post-treatment clients associated the people in the picture with drug use, they reacted more negatively. They characterized them as dumb, crazy, 
Pre-treatment

Total Score: 1931

Total Score: 1811

PRE-TREATMENT: These clients are more specific

mentioning different types of drugs and alcohol. Crack and cocaine are the drugs referred to the most.
POST-TREATMENT: These clients express stronger feelings about drugs. While they think of positive effects, like being high and having fun, they focus on negative evaluations: drugs cause pain, both physical and mental, and they are dangerous. They pain, both physical and mental, and they are dangerous. They

also refer to drugs as a sickness and talk about treatment.

Furthermore, they associate drugs with feelings of loneliness,

misery, and depression, and they express fear of drugs. They also denounce drugs as bad, negative, and insane.

Figure 1. Perceptions and evaluations of drugs by pre-treatment and post-treatment clients. 


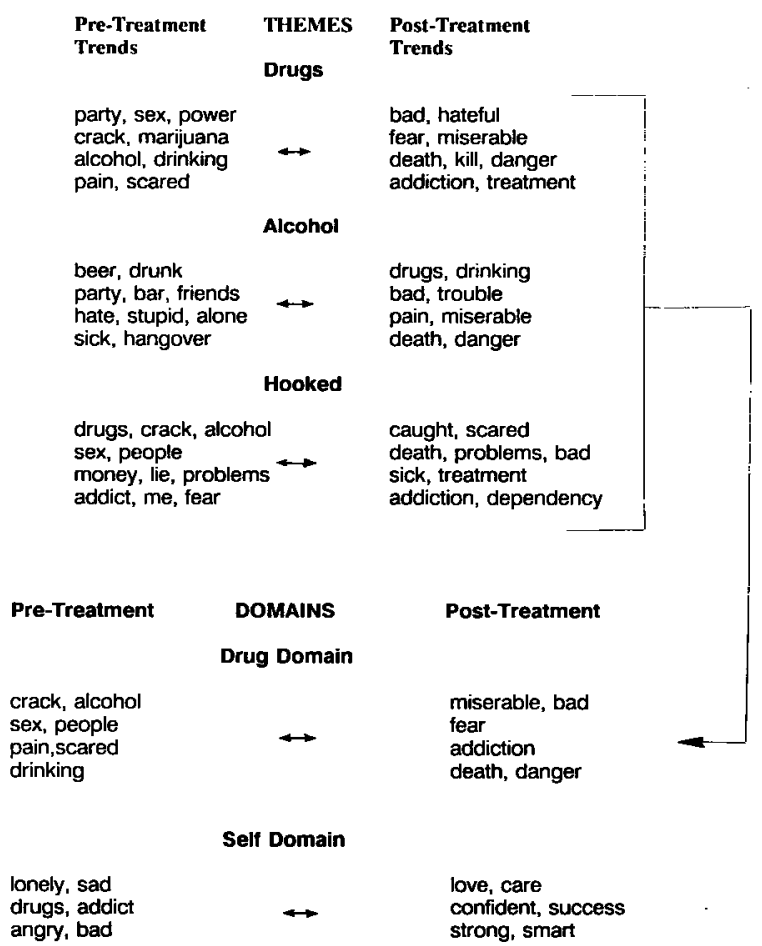

Figure 2. Dominant trends of perceptions and evaluations differentiating pre-treatment from post-treatment clients.

and bad. In response to pictures that showed people who appeared as troubled or depressed, the post-treatment clients thought more about helping, talking, and understanding. They also thought more of friends, indicating the tendency to view friends as instrumental in solving or coping with personal problems; they may rely on friends more for support.

It is apparent that compared to the pre-treatment clients, the post-treatment clients have a more detailed and more advanced view of drugs. They have a more negative opinion of drugs, and they are more sensitive to and critical of their potential role. In general, compared to pre-treatment addicts, whose thoughts are more limited to the concrete and visible, the post-treatment group shows an increased ability to penetrate the surface of concrete details and look for deeper meanings involving human feelings and interpersonal relationships. The response trends observed across the thirty pictures used in these investigations suggest that the treatment program has had an impact on the way in which these people look at life events and on how they try to cope with them.

\section{Identification of Rehabilitated Clients}

The finding of distinct differences between the pre-treatment and post-treatment groups in their perceptions and attitudes suggested an opportunity to test to what extent these response patterns would allow us to determine whether an individual client belonged to a pre-treatment or posttreatment group. Discriminant function analysis of the clients' individual dominance, evaluative, and perceptual similarly scores based on their free associations to 10 selected pictures resulted in correct identification of $91 \%$ of the clients as having received treatment or not (see Figure 4). These results are in line with our earlier studies of $\mathbf{4 0 0}$ clients in treatment (Szalay, in press) and 120 clients in treatment (Szalay et al., in press), all showing that free associations are clearly sensitive measures of changes in the clients' psychological dispositions: perceptions, priorities, and attitudes.

\section{Discussion}

Free associations provide the mosaic elements of subjective images and offer heretofore little 
PERCEPTIONS AND EVALUATIONS

BY

Pre-treatment

Post-treatment

Total Score: 1195

Total Score: 1376

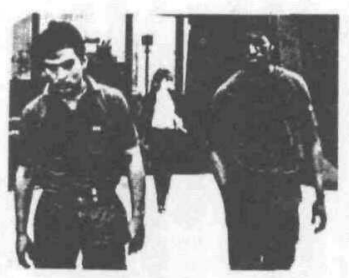

\begin{tabular}{lrr} 
& PRE & PST \\
JOB, JOBLESS & 202 & 139 \\
\hline unemployment & 30 & 26 \\
work & 31 & 43 \\
job, s & 11 & 26 \\
jobless & - & 15 \\
no job & 37 & $:$ \\
fired & 22 & $:$ \\
job hunt ing & 12 & $:$ \\
money & 22 & 21 \\
bankrupt, cy & 11 & $:$ \\
poor, ness & 18 &. \\
broke & 8 & 8
\end{tabular}

DEPRESSION $(23 \%, 21 \%)$

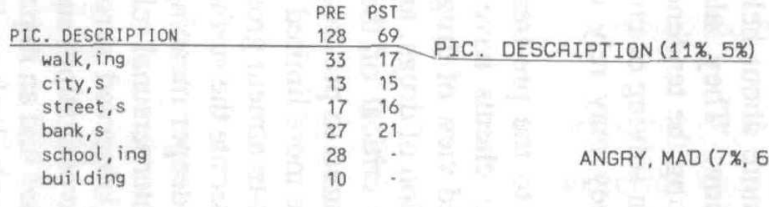

ORUGS, ADDICTS $(5 \%, 5 x)$

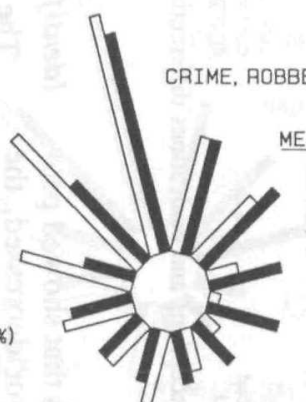

FIGHTING, VIOLENCE (2\%, 5\%)

MEN, FRIENDS $(8 \%, 10 \%)$

TROUBLE, PAOBLEMS $(3 \%, 7 \%)$

$\begin{array}{ll}\text { FIGHTING, VIOLENCE (2\%, 5\%) } & \text { help, ing, } \\ \text { PEJECTION }(4 \%, 5 \%) & \text { cope, ing } \\ \text { care, ing }\end{array}$
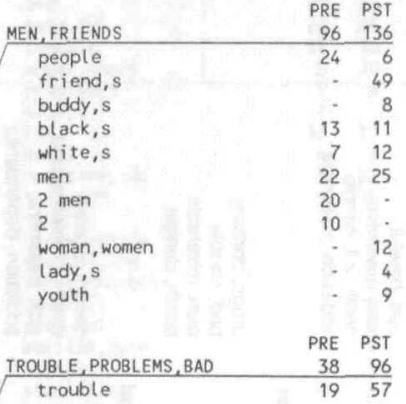

trouble

bad

11. $\quad 8$

no good

sick, ness

disgust, ing

CARE, HELP, COPE

PRE PST

help, ing, ed

$11 \quad 92$

concern, ed

hope, ful

accept, ance
support, ive

PRE-TREATMENT: These clients see two men who are

unemployed and looking for work. They refer more to

POST-TREATMENT: These clients think the two men are friends. They see them as having problems but they also overwhelmingly think of helping, coping and support.
They focus on solving or coping with the problems and

physical aspects of the scene, such as the building the they rely on friends to be there to help them.

Figure 3. Perceptions and evaluations of picture \#918 by pre-treatment and post-treatment clients. 


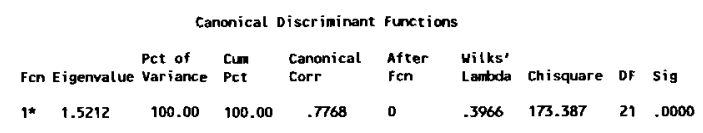

- marks the 1 canonical discriminant functions remaining in the analysis.

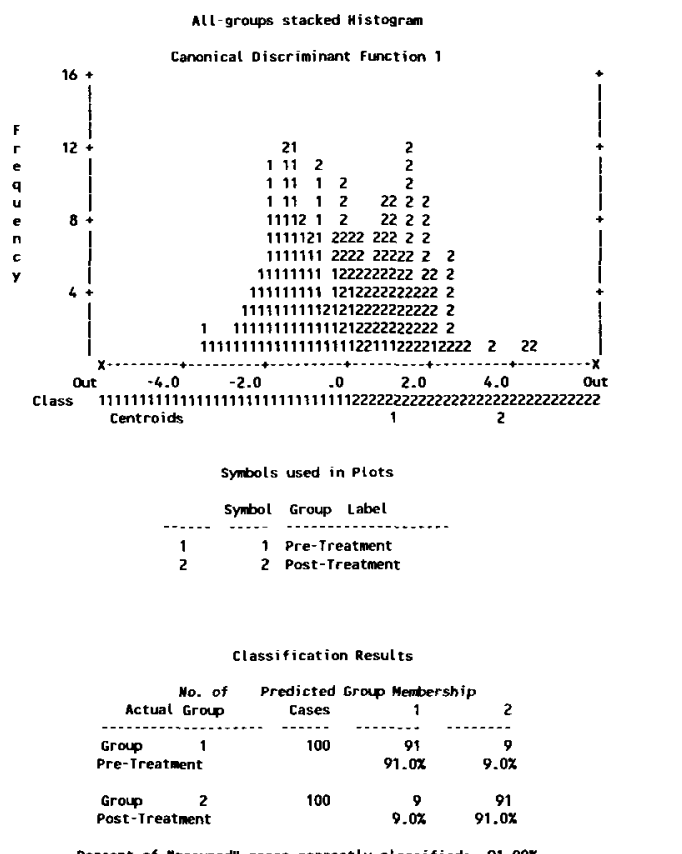

Figure 4. Canonical discriminant function differentiating pretreatment from post-treatment clients.

recognized opportunities for the reconstruction and mapping of subjective worlds based on a solid empirical foundation. The representational approach used in the investigations reveals trends in the perceptual and motivational dispositions that are dominant in the drug user's subjective world. The dominant elements of subjective representation are the ones most likely to affect behavior and therefore deserve special attention. In the context of drugs, for example, recognition of potential harm and danger associated with drug abuse is stronger for post-treatment clients than for pre-treatment clients. This recognition can be an important factor in preventing them from relapsing.

Assessing perceptual and motivational trends requires more than asking "Do you think drugs are harmful?"' Responses to such questions tend to reflect conscious judgments rather than what is actually salient in the respondent's subjective world. The representational approach is an effective strategy for accessing the salient perceptions and attitudes characteristic of drug abusers in various stages of addiction. The attention paid to diverse personal, psychological, health, social, economic, and legal consequences of drug use has been found to vary among different treatment groups (Szalay et al., in press), student groups (Szalay, Strohl \& Wilson, 1991; Szalay, 1990), and culture groups (Szalay, Canino \& Vilov, in press).

Some of the applications discussed may be criticized for various limitations, for example, the reliance on a cross-sectional design rather than a longitudinal design in program evaluation. The main purpose of the present article, however, is to call attention to the value of free associations in clinical applications in which the researcher is likely to have his/her own methodological preferences.

\section{The Relative Value of Word-and Picture- stimulated Associations}

The method of word-stimulated free associations, which was illustrated briefly in this paper, has been used in dozens of in-depth studies of groups representing select cultures, subcultures, and cohorts of different social strata and behaviors (e.g., drug users and non-users). As these results have demonstrated, this approach offers a sensitive analytic method to assess subjective images and meanings and to reconstruct people's subjective worlds from the mosaic pieces. The AGA method offers an advanced tool for the ambitious research objective often called "cognitive mapping." Since this word association based assessment goes beyond the perceptual/cognitive dimensions to include affective/motivational dimensions, the mapping may be better characterized as the reconstruction of people's systems of subjective representation. The importance of this system follows from its role to provide direction and organization for behavior.

Just as our world encompasses numerous domains, such as family, work, and religion, this analysis can be conveniently performed by reconstructing the subjective representation of these domains (e.g., the domain of family) from the images (e.g., father, mother, relatives) that form the mosaic pieces. Then, from several domains (e.g., family, work), the subjective world of the addict can be reproduced in areas relevant to research interest. This rather remarkable analytic 
capability is at the very core of the word stimulated association method which reveals how a particular person or group perceives and evaluates certain stable elements of their environment.

Picture-stimulated associations reveal perceptual and evaluative trends that are more context free. We found, for instance, that addicts prior to treatment respond rather superficially and concretely to the pictures, that is, they basically describe the concrete things depicted and show little interest and/or capability for a deeper analysis or interpretation of these stimuli. These concrete perceptions indicate that the pre-treatment addicts either are disinclined to expend much effort for the task or they have only a limited capability to grasp the deeper meanings of social situations and relationships therein depicted. Post-treatment addicts become increasingly sensitive to the importance of interpersonal relations; they show more empathy and more readiness to recognize human problems. They also place more emphasis on friendship and a willingness to help.

A combination of word and picture stimuli promises a rich resource of information on the psychological disposition of drug abusers. The word-stimulated associations offer insights into perceptual and attitudinal dispositions differentiating pre/post-treatment populations in select themes and domains, such as drugs, self, and interpersonal relationships. The picture-stimulated associations reveal some characteristic differences in the level and style of cognitive/perceptual processes characteristic of these two treatment populations.

The psychological dispositions traced by both the word- and picture-stimulated free associations offer new opportunities for monitoring the psychological effects of the treatment process, as well as a new empirical measure of program success.

\section{Therapeutic Applications}

The new information produced on subjective images and meanings relates to several applications ranging from improved communications to therapy.

Improving communications with the client. Information on the subjective images and meanings of pre-treatment and post-treatment clients can have many useful applications in therapeutic communications. Familiarity with people's subjective priorities and meanings is probably the most critical prerequisite for good rapport and effective communication. Past communication studies with
AGA-based information has conclusively shown that messages built on people's subjective images and meanings (reconstructed from their free associations) were judged as more meaningful and cogent than messages drawn from other sources, such as experts and professionals (Szalay, Lysne, \& Bryson, 1972).

Treatment of addicts. The new findings show first of all a heretofore little recognized close relationship between drug use and psychological dispositions. In the present study, and in other comparable studies (Szalay, 1991; Szalay et al., in press), pre-treatment and post-treatment clients were found to show such consistent differences related to treatment effects. Another series of studies comparing drug users and non-users (Szalay, 1990, Szalay et al., 1991; Szalay et al., 1991) have also revealed similar perceptual and motivational dispositions significantly related to drug use.

The assessment of internal states associated with drug use provides new opportunities for measuring the severity of addictions. Their use in treatment offers new opportunities to trace changes within the course of the treatment process. The new information suggests new insights into the black box of the internal states of individual clients, as well as into the dark tunnel of the treatment process.

Just as the substance abuse treatment field desperately needs relevant, reliable, and valid measure of treatment outcome (Carroll, in press), so too does the field need process measures of treatment progress, stagnation, and regression. The Associative Group Analysis (AGA) technique promises new opportunities for feedback on the effectiveness of the treatment process based on its psychological effects on the addict. In a treatment world distinguished by utilization and review requirements and greater accountability for treatment program evaluations, devices such as the AGA would be most beneficial to both administration and practitioners.

Uses in psychotherapy. New in-depth information on subjective meanings naturally bears on therapeutic interests in the inner world of the client. This interest has a particularly strong foundation in schools with a cognitive orientation, such as cognitive behavior therapy (Beck, 1976) and rational-emotive therapy (Ellis, 1962; 1975). These schools not only recognize the difference between subjective meanings and reality, but also consider this difference to be a main factor in depression and neuroses. From this angle, a main 
objective of the therapeutic process is to bring the client's subjective meanings closer to those of his/her environment.

As recent findings show, cognitive/behavioral therapies have been successful in affecting behavior change by altering the client's cognitions and meanings toward a greater degree of conformity with reality. Diverse applications of this therapeutic approach are evident in the alleviation of anxiety and distress (Bandura, 1986), the treatment of bulemia and anorexia nervosa (Fairburn, 1990), and the treatment of alcoholism (Adams \& McNeal, 1991; Johnson, 1991). Common to most of these interventions is the recognition that the client's perceptions differ from reality. The success of therapists depends naturally on a valid assessment of these differences, on the identification of the pathogenic forces - the misperceptions, unrealistic expectations, and mistaken beliefs-at the source of various emotional and behavioral disorders. It is here that AGA offers new insights. The utility of this information in psychotherapy with addicts will vary greatly depending on the pathogenic dispositions and their accessibility by the therapist.

As indicated, therapists employ various techniques to assess the subjective world of addicts, mainly by talking and asking questions. Based on a long tradition, there is a tacit assumption that the experiences and skills developed in this process may be the only genuine resources available. While the value of the more traditional therapeutic communication is not at question, free associations are shown to provide rich additional sources of information.

AGA is a versatile tool which can be used at several levels. Compared with the advanced tools of cognitive mapping, most of the clinical applications of AGA can be simple and non-technical. Just as Freud used word associations without statistics and without computers, the comparison of simple response distributions obtained from individual clients or their cohorts (i.e., drug addicts) can provide useful insights at a relatively nontechnical level. While tests for specific diagnostic application are being developed, the authors will be glad to share their experiences to assist colleagues in their interest to adapt AGA to their individual needs.

\section{References}

Amas, S. L. \& McNeIL, D. W. (1991). Negative alcohol expectancies reconsidered. Psychology of Addictive Behavior, 5, 1, 9-14.
BANDURA, A. (1986). Social foundations of thought and action: A social cognitive theory. Englewood Cliffs, NJ: Prentice-Hall.

BANDleR, J. \& GRINDER, R. (1982). Re-framing-Neurolinguistic programming and the transformation of meaning. Moab, Utah: Real People Press.

Beck, A. T. (1976). Cognitive therapy and the emotional disorders. New York: New American Library.

BIASE, D. V. (1984). A drug abuse prevention program developed within an urban T.C. Journal of Psychoactive Drugs, 16(1), 63-68.

Biggs, S. J., Bender, M. P., \& Foreman, J. (1983). Are there psychological differences between persistent solvent abusing delinquents and delinquents who do not use solvents? Journal of Adolescence, 6(1), 71-86.

Botvin, G. \& MCAluster (1982). Cigarette smoking among children and adolescents: Causes and prevention. In C. Arnold (Ed.), Annual review of disease prevention. New York: Springer.

Burr, A. (1984). The ideologies of despair: A symbolic interpretation of punks and skinheads usage of barbituates. Social Science \& Medicine, 19(9), 929-938.

CarroLL, J.F.X. (1982). Personality and psychopathology: A comparison of alcohol and drug-dependent persons. In J. Solomon and K. A. Keeley (Eds.), Perspectives in alcohol and drug abuse: Similarities and differences (pp. 5988) Boston: John Wright PSG.

Carroul, J.F.X. (in press). Clinical issues in therapeutic communities. National Institute of Drug Abuse: Research Monograph Series.

CRAIG, R. J. (1986). The personality structure of heroin addicts. National Institute of Drug Abuse: Research Monograph Series, monograph 74, 25-36.

Craig, R. J. (1988). Psychological functioning of cocaine free basers and derived from objective psychological tests. Journal of Clinical Psychology, 44(4), 599-606.

DEESE, J. (1965). The structure of associations in language and thought. Baltimore: John Hopkins University Press.

Deleon, G. (1986). Therapeutic community: Perspective and approach. In G. DeLeon and J. T. Ziegenfuss (Eds.), Therapeutic communities for addictions: Readings and theory, research and practice. Springfield, IL: Charles C Thomas.

Delleon, G. \& Rosenthal, M. S. (1989). Treatment in residential therapeutic communities. In T. B. Karasu (Ed.), Treatments of psychiatric disorders (Vol. 2) (pp. 13791396). Washington, DC: American Psychiatric Association.

DES JARLAIS, D. C. (1986). Locus of control and need for control among heroin users. National Institute of Drug Abuse: Research Monograph Series, monograph 74, 37-44.

Dielman, T. E., Campanelli, P. C., Shope, J. T., \& Butchart, A. T. (1987). Susceptibility to peer pressure, self esteem, and health locus of control as correlates of adolescent substance abuse. Health Education Quarterly, 14(2), 207-221.

Doherty, O. \& Matrhews, G. (1988). Personality characteristics of opiate addicts. Personality and Individual Differences, $9(1), 207-221$.

DOWNs, R. M. \& STEA, D. (1973). Image and environment: Cognitive mapping and spacial behavior. Chicago: Aldine.

EuLs, A. (1962). Reason and emotion in psychotherapy. New York: Lyle Stuart.

Euls, A. \& MuRPHY, R. (1975). A bibliography of articles and books on rational-emotive therapy and cognitive-behavior therapy. New York: Institute for Rational Living.

FaIrburN, C. G. (1990). Treatment modalities for eating dis- 
orders. Paper presented at the Bulimia Anorexia Self-Help Eighth Intemational Conference on the Eating and Mood Disonders, April.

FrankL, V. E. (1962). Man's search for meaning: An introduction to logotherapy. New York: Simon and Schuster.

Jornsion, P. B. (1987). Reaction expectancies and ethnic drinking differences. Psychology of Addictive Behavior, 1 , 5, 36-40.

JuRICH, A. P. Polson, C. J. (1984). Reasons for drug use: Comparison of drug users and abusers. Psychological Reports, 55(2), 371-378.

KanDEL, D. B. (1980). Drug and drinking behavior among youth. Annual Review of Sociology, 6, 235-285.

KAPLAN, H. B. (1982). Self-attitudes and deviant behavior: New directions for theory and research. Youth and Society, 14(2), 185-211.

Krampen, G. \& Von EYe, A. (1984). Generalized expectancies of drug delinquents, other delinquents, and a control sample. Addictive Behaviors, 9(4), 421-423.

Malvin, J. H., Moskowitz, J. M., Schaps, E., \& SchaefFER, G. A. (1985). Evaluation of two school based alternative programs. Journal of Alcohol and Drug Education, 30(3), 98-108.

McBriDE, K. J. (1982). I-E scores of drug abusing offenders: The influence of social desirability. Criminal Justice and Behavior, 9(2), 177-183.

Miller, G. A. (1967). Psycholinguistic approaches to the study of communication. In D. Arm (Ed.), Journeys in science. Albuquerque: University of New Mexico Press.

Noble, C. (1952). An analysis of meaning. Psychology Review, 54, 421-440.

Olton, A. L. (1985). The effect of locus of control and perceptions of school environment on outcome in three school drug abuse prevention programs. Journal of Drug Education, 15(2), 157-169.

Osgood, C. E., Suci, G. J., \& Tannenbaum, P. H. (1957). The measurement of meaning. Urbana: University of Illinois Press.

Rohsenow, D. J., Smith, R. E., \& Johnson, S. (1985). Stress management training as a prevention program for heavy social drinkers. Addictive Behaviors, 10(1), 45-54.

SEgal, B. \& Merenda, P. F. (1975). Locus of control, sensation seeking, and drug and alcohol use in college students. Drug Forum, 4(4), 349-369.

ShFDlfr, J. \& Block, J. (1990). Adolescent drug use and psychological health: A longitudinal inquiry. American Psychologist, 45, 612-630.

Shepard, R. M. \& ChIPMAN, S. (1970). Second order isomorphism of internal representations: Shapes of state. Cognitive Psychology, 1, 1-7.

Stran, K. B., Soskin, W. F., \& Korchin, S. J. (1975). Drug use among disaffected high school youth. Journal of Drug Education, 5(3), 193-203.

Sterman, C. M. (1991). Neuro-linguistic programming as psychotherapeutic treatment in working with alcohol and other drug addicted families. Chemical dependency: Theoretical approaches and strategies, 73-85.
Sutker, P. B. \& Aluian, A. N. (1988). Issues in personality conceptualizations of addictive behaviors. Journal of Consulting and Clinical Psychology, 56(2), 172-182.

SzAl_AY, L. B. (1990). Institutional climate and substance abuse: A multidimensional assessment. Chevy Chase, MD: Institute of Comparative Social and Cultural Studies.

Szalay, L. B. (in press). Socialization into the T.C. culture. In G. DeLeon and F. Tims (Eds.), Therapeutic community research. National Institute of Drug Abuse Monograph.

Szalay, L. B. \& Brent, J. (1967). The analysis of cultural meanings through free verbal associations. Journal of Social Psychology, 72, 161-187.

Szalay, L. B., Bovasso, G. Vilov, S. K. \& Whliams, R. E. (1992). Assessing treatment effects through changes in perceptions and cognitive organization. American Journal of Drug and Alcohol Abuse, 18(4), 407-428.

Szalay, L. B. \& Bryson, J. A. (1973). Measurement of psychocultural distance: A comparison of American Blacks and Whites. Journal of Personality and Social Psychology, 26, 166-177.

Szalay, L. B. \& Bryson, J. A. (1974). Psychological meaning: Comparative analyses and theoretical implications. Journal of Personality and Social Psychology, 30, 860-870.

Szalay, L. B., Canino, G. , \& Vilov, S. K. (1993). Vulnerabilities and culture change: Drug use among Puerto Ricans in the United States. The International Journal of the Addictions, 28, 327-354.

Szalay, L. B. \& Deese, J. (1978). Subjective meaning and culture: An assessment through word associations. Hillsdale, NJ: Lawrence Erlbaum/Wiley.

Szalay, L. B., Inn, A., Strohl, J. B. \& Wilson, L. C. (in press). Perceived harm, age, and drug use: Perceptual and motivational dispositions affecting drug use. Journal of Drug Education.

Szalay, L. B., Lysne, D. A., \& Bryson, J. A. (1972). Designing and testing cogent communications. Journal of Cross-Cultural Psychology, 3, 247-258.

Szalay, L. B., Strohl, J. B., \& Wilson, L. C. (1991). Adolescent socialization, changing views related to drug use. Chevy Chase, MD: Rapport.

Szalay, L. B., Windle, C., \& Lysne, D. A. (1970). Attitude measurement by free verbal associations. Journal of Social Psychology, 82, 43-55.

Tolman, E. C. (1948). Cognitive maps in rats and men. Psychology Review, 55, 189-208.

Triandis, H. C. \& Vassiliou, V. (1967). Componential analysis of subjective culture. Urbana, IL: University of Illinois Press.

Wemman, A. (1983). The compulsive adolescent substance abuser: Psychological differentiation and family process. Journal of Drug Education, 13(2), 161-172.

Zeiner, A. R. Stanitis, T., Spurgeon, M., \& Nichols, N. (1985). Treatment of alcoholism and concomitant drugs of abuse. Alcohol, 2(3), 555-559. 